\title{
El léxico de la viruela en la Guatemala del siglo XVIII: algunas notas sobre la Instrucción sobre el modo de practicar la inoculación de las viruelas de José Felipe Flores (Ciudad de Guatemala, 1794)
}

\author{
Smallpox Lexicon in 18th Century Guatemala: Some Notes about Instrucción \\ sobre el modo de practicar la inoculación de las viruelas, by José Felipe Flores \\ (Guatemala City, 1794)
}

JosÉ LUIS RAmírez LUENGo [joseluis.ramirezluengo@gmail.com]

Universidad Autónoma de Querétaro, México

\begin{abstract}
RESUMEN
Mientras que el léxico médico dieciochesco ha sido notablemente estudiado en el caso de España y de algunas zonas americanas como México, en otras áreas como Guatemala constituye un tema que hasta el momento ha recibido muy poca o nula atención por parte de los estudiosos; precisamente por esto, el presente trabajo pretende aportar una primera descripción del vocabulario relacionado con la viruela que es usado en la Instrucción sobre el modo de practicar la inoculación de las viruelas, tratado del doctor José Felipe Flores que se publica en 1794 en la Ciudad de Guatemala. De este modo, los objetivos fundamentales del análisis serán tres: a) extraer los vocablos relacionados con la viruela presentes en el texto indicado; b) señalar las coincidencias y divergencias existentes en este léxico entre diversas zonas de la Nueva España, y más en concreto Guatemala y la Ciudad de México; y c) discutir posibles líneas de investigación que contribuyan a un mejor y más profundo conocimiento de la historia del vocabulario médico utilizado en el antiguo Reino de Guatemala.
\end{abstract}

\section{Palabras Clave}

Historia del español de América; Guatemala; léxico; viruela; siglo XVIII

\begin{abstract}
Although 18th-century medical lexicon has been studied in Spain and some American areas like Mexico, in the case of Guatemala this subject has received no attention from scholars. Because of that, this paper aims to provide the first description of lexicon related to smallpox that is used in Instrucción sobre el modo de practicar la inoculación de las viruelas, a treatise published by doctor José Felipe Flores in 1794 in Guatemala City. Thus, the purposes of this analysis are three: a) to list lexical items related to smallpox which appear in the corpus; b) to point out coincidences and divergences in this lexicon that can be discovered between Guatemala and other areas of New Spain such as Mexico City, and c) to discuss new lines of research that contribute to improving our knowledge of the history of the medical lexicon that is used in the old Kingdom of Guatemala.
\end{abstract}




\section{KEYWORDS}

History of Latin American Spanish; Guatemala; lexicon; smallpox; 18th century

RECIBIDO 2020-04-20; ACEPTADO 2020-08-23

1. No resulta novedoso indicar que, mientras que el léxico médico dieciochesco ha sido notablemente estudiado en el caso de España (entre otros, Gómez de Enterría 2012a, 2012b, 2013; Gómez de Enterría y Gallardo 2010; Gutiérrez Rodilla 1993, 1994-5, 1996), América se muestra como una zona bastante más inexplorada al respecto, por más que últimamente se hayan desarrollado ya algunas investigaciones que, desde diferentes puntos de vista, ofrecen cierta información acerca de Colombia (Parcero Torre 2011), Argentina (Ramírez Luengo 2015b) y de forma más abundante el actual México (Gómez de Enterría 2014; Ramírez Luengo 2014, 2015, 2019, 2020); se puede decir, por tanto, que el estudio del vocabulario de esta ciencia en el Nuevo Mundo no es el hueco en blanco que era hasta hace poco tiempo, pero no cabe duda que sigue siendo una temática sobre la que es necesario profundizar aún más, especialmente en el caso de regiones del continente a las que por el momento no se ha prestado la atención que merecen.

Dentro de estas regiones poco o nada estudiadas desde el punto de vista de la lexicología, es sin duda importante señalar el caso del antiguo Reino de Guatemala ${ }^{1}$ y muy especialmente de su capital colonial, Santiago de los Caballeros -hoy Antigua Guatemala-, en cuya Real y Pontificia Universidad de San Carlos se desarrolla desde muy pronto el estudio de la medicina: en efecto, tal y como señalan diferentes autores (Asturias 1902: 214; Álvarez Sánchez 2016: 20), a los pocos meses de la fundación de la institución académica, en 1681, se inician estos estudios a cargo del Doctor Miguel Fernández, y a pesar de que la cantidad de alumnos inscritos en ningún caso es abundante, lo cierto es que ya para los primeros años del siglo XVIII se cuenta con los primeros graduados; esta situación se mantiene sin cambios hasta la época de la Independencia, si bien esto no impide que se produzca cierto florecimiento de la disciplina durante el Siglo de las Luces, algo que se hace patente tanto en la aparición del Protomedicato guatemalteco (Asturias 1902: 222) como en el prestigio que adquieren algunos galenos que ejercen en la región -entre otros, José Antonio Córdova, Narciso Esparragosa y Gallardo o el mismo José Felipe Flores- y, a raíz de lo anterior, en la publicación de cierto número de obras de esta temática en la actual capital del país².

1 Existen, por supuesto, trabajos de índole histórica sobre la cuestión, entre los que se pueden citar las obras de referencia de Asturias (1902) y Martínez Durán (2010) sobre el desarrollo general de la medicina en la región o artículos puntuales sobre la presencia de esta disciplina en la Gaceta de Guatemala (Hernández Pérez 2010) o sobre la expansión de la vacuna (Smith 1974).

2 Entre otras -y más allá del texto que se va a estudiar en estas páginas-, cabe citar la Memoria sobre una invención fácil y sencilla para extraer las criaturas clavadas en el paso sin riesgo de su vida (Nueva Guatemala: Ignacio Beteta, 1798), de Narciso Esparragosa y Gallardo, o el Especifico nuevamente descubierto en el Reino de Goatemala, para la curación radical del horrible mal del cancro (Nueva Guatemala: s.i., 1781), del mismo José Felipe Flores, así como los Rudimentos físico-canónico-morales o glosa al edicto del Ylustrisimo señor Don Cayetano Francos y Monroy (Nueva Guatemala: Viuda de Sebastián de Arévalo, 1786) de Pedro José de Arrese, texto no estrictamente médico pero en el que se tratan aspectos relacionados con la ginecología; ya en los primeros años del siglo XIX aparece la Cartilla que enseña el modo de vacunar $y$ distinguir la verdadera y falsa vacuna (Nueva Guatemala: s.i., 1804?), de Juan Antonio Córdova, o el Reglamento para la propagación y estabilidad de la vacuna en el Reyno de Guatemala (Nueva Guatemala: Ignacio Beteta, 1805), todo lo cual da muestras de la importancia que adquiere la medicina en la producción impresa del país. 
Teniendo en cuenta, por tanto, lo que muy brevemente se acaba de señalar sobre el desarrollo de esta ciencia de la Guatemala ilustrada, sorprende que hasta el momento no se haya llevado a cabo el estudio del léxico médico empleado en la región, muy especialmente si se tiene en cuenta la importancia que esta cuestión puede tener no solo por sí misma, sino muy especialmente para conseguir una comprensión más profunda de la situación que se descubre al respecto en otras zonas de América y, a partir de aquí, de los procesos que van a terminar por configurar la moderna terminología médica en el español.

2. En línea con lo que se acaba de señalar, el presente trabajo pretende ser una primera aproximación al vocabulario de la medicina -y más en concreto de la viruela- que aparece en la Instrucción sobre el modo de practicar la inoculación de las viruelas, obra muy breve, de poco más de quince páginas, que redacta el doctor José Felipe Flores y que es publicada en las postrimerías del siglo XVIII en Nueva Guatemala de la Asunción, la actual Ciudad de Guatemala.

Por lo que se refiere al autor, no cabe duda de que se trata de la personalidad más importante de la medicina centroamericana colonial (Asturias 1902: 220)3 : según señalan Belaubre (2019) y Barona Vilar (2019), José Felipe Flores nace en Ciudad Real de Chiapas en 1751, y aunque sus primeros años de vida son prácticamente desconocidos, se sabe que se traslada pronto a Santiago de los Caballeros, donde en 1773 obtiene el título de bachiller en medicina por la Universidad de San Carlos; unos años después, trabajando ya como médico en el Hospital de San Juan de Dios, se hace cargo de la cátedra de esta especialidad en la mencionada universidad, y tanto en esta como en el hospital comienza a incorporar importantes reformas desde el punto de vista educativo y asistencial, colaborando en la formación de futuros médicos, construyendo modelos anatómicos de cera de notable calidad y generalizando la práctica de la inoculación como respuesta a la epidemia de viruela que en 1780 se extiende por el reino.

Por supuesto, todas estas cuestiones no hacen sino acrecentar su fama en los círculos profesionales, lo que tiene su reflejo en hechos como que en 1793 sea nombrado responsable del Protomedicato guatemalteco que él mismo ha impulsado o que la comisión española que está planeando la Real Expedición Filantrópica de la Vacuna tome en consideración su experiencia y sus informes científicos para poner en marcha la iniciativa ${ }^{4}$; algo más tarde, en 1797, se embarca en un largo viaje que, con el objetivo de ampliar sus conocimientos, lo lleva a Cuba, Estados Unidos, el norte de Europa, Italia y finalmente España, en cuya capital se establece desde 1800 y donde, designado médico de cámara del rey Fernando VII, termina por fallecer en 1814.

En cuanto al texto en sí -que presenta el título de Instrucción sobre el modo de practicar la inoculación de las viruelas y método para curar esta enfermedad, acomodada a la naturaleza y modo de vivir de los indios del Reyno de Guatemala-, hay que señalar que es una de las dos únicas obras conocidas del doctor Flores ${ }^{5}$; como se dijo anteriormente, se trata de un opúsculo muy breve, de

3 Dado que no es este el objeto del trabajo, se aportan únicamente unos primeros datos sobre la vida del autor; para una biografía completa y pormenorizada del doctor chiapaneco, véase la obra de Aznar López (1998).

4 Sobre estas dos cuestiones, véanse los trabajos de Rodríguez (1990) y Galich (1982) respectivamente, donde se analizan ambos aspectos de forma monográfica.

5 Según se ha indicado ya, la otra, de mayor difusión en su época, se denomina Especifico nuevamente descubierto en el Reino de Goatemala, para la curación radical del horrible mal del cancro y otros más frecuentes, y no solo se publica en la capital guatemalteca, sino también en Ciudad de México, Madrid y Cádiz, así como -traducida al francés y al italiano- en 
17 páginas en cuarto, que se publica en 1794 en la Imprenta de Ignacio Beteta, en la Ciudad de Guatemala (López Piñero et alii. 1992: 99), y que se compone de dos partes claramente diferenciadas: una primera, que constituye la Instrucción en sí misma (pp. 3-10), y una segunda que se compone de una serie de documentos de naturaleza administrativa -en concreto, varios memoriales del mismo Flores explicando las decisiones adoptadas para evitar el contagio de la viruela o un certificado del escribano de cámara autorizando el desembolso económico necesario para la publicación del texto- que resultan de interés porque permiten conocer las vicisitudes burocráticas que preceden a su publicación (pp. 11-17).

Pasando ahora a su contenido, es importante mencionar que el hecho de que sea una obra pensada no tanto -o no exclusivamente, al menos- para especialistas determina tanto las temáticas presentes en ella como su propia organización interna ${ }^{6}$ : así, si bien el texto comienza con una definición de la inoculación (pp. 3-4), rápidamente se pasa a describir el método para llevarla a cabo (pp. 4-5) y diversas cuestiones que favorecen la lucha contra la viruela, tales como los cuidados necesarios en el caso de enfermos específicos, el régimen alimenticio que se ha de respetar (pp. 5-9) o las precauciones que se han de seguir para evitar el contagio entre municipios (pp. 9-10). Salta a la vista, por tanto, que se trata de una obra eminentemente práctica ${ }^{7}$ que está pensada para que, desde las instancias del gobierno colonial, se evite la propagación de la viruela y en la medida de lo posible se puedan paliar los efectos de la epidemia, circunstancia que sin duda es necesario tener en cuenta para poder valorar de una manera más adecuada el léxico que se registra en sus páginas.

Teniendo en cuenta, pues, todo lo indicado hasta el momento, parece posible fijar los objetivos fundamentales de este análisis en cuatro: a) extraer los vocablos relacionados con la medicina $-\mathrm{y}$ más específicamente con la viruela- presentes en el texto indicado; b) señalar las coincidencias y divergencias existentes en este léxico entre diversas zonas de la Nueva España, en concreto Guatemala y el Altiplano mexicano (Ramírez Luengo 2014); c) de forma secundaria, detectar la presencia de voces populares (Ramírez Luengo 2015) en el discurso médico guatemalteco del Siglo Ilustrado relacionado con esta dolencia; y d) apuntar posibles líneas de investigación que contribuyan en un futuro próximo a un mejor y más profundo conocimiento de la historia del vocabulario de la medicina que es utilizado durante el Setecientos en el antiguo Reino de Guatemala.

\footnotetext{
Lausana, Turín y Venecia (López Piñero et alii. 1992: 97-98); aunque pueda sorprender tan escasa producción en un autor tan prominente, esto se debe, en palabras de Barona Vilar (2019), a que "Flores era más bien un hombre de acción que un escritor reflexivo", algo que queda demostrado no solo por la ya mencionada escasez de obras científicas debidas a su pluma, sino también por el claro contenido práctico de estas, tal y como se indicará más adelante.

6 El mismo título -y en concreto su referencia al hecho de estar acomodada "a la naturaleza y modo de vivir de los indios" (p. 1)- demuestra ya que aquellos a quienes va destinada no son exclusivamente los profesionales de la medicina, idea que se ve apoyada, además, en las múltiples referencias que aparecen en el texto a las labores que, para la mejor prevención de la viruela, tienen que desarrollar ciertos miembros de la administración como son los principales, las autoridades municipales o incluso los sacerdotes (pp. 3, 9, 10, 12).

7 Este carácter eminentemente práctico es puesto de manifiesto por el propio Flores, quien en uno de los memoriales que se incorporan al impreso señala que "habiéndose tenido presentes la variedad de clima de las provincias, la pobreza, rusticidad y modo de vivir de los indios y demás castas, la falta de socorros y arbitrios en los pueblos (...) he formado una instrucción sencilla y clara y acomodada al régimen de los habitantes y producciones de sus países, la que presento a V. S. para que teniéndolo a bien, mande imprimirla" (p. 11).
} 
3. Se ha señalado ya en numerosas ocasiones (Rodríguez 2001; Cortés Riveroll 2008) que a lo largo del siglo XVIII la viruela se convierte en una cuestión médica de primera importancia en el ámbito de la Nueva España, cuestión a la que, naturalmente, Guatemala no permanece ajena: en efecto, la aparición de diversas epidemias a lo largo del siglo -en concreto, en 1733, 1780 y 1794 (Asturias 1902: 125, 130) - y la necesidad de detener el contagio entre las diversas zonas del Reino, tanto con medidas de cuarentena como con la práctica de la inoculación a partir de 1780 (Asturias 1902: 126, 137-138, 140), determina la publicación de numerosos escritos que pretenden luchar contra esta dolencia y en los que se hace uso de un vocabulario muy abundante relacionado con diferentes aspectos de la enfermedad; no sorprende, por tanto, que en el texto analizado en estas páginas se presente una enorme riqueza de unidades léxicas referidas a diferentes cuestiones como, entre otras, la nomenclatura de los tipos de viruela, la denominación aplicada al enfermo o las etapas de desarrollo de la misma.

Por lo que toca al nombre de la enfermedad, es de destacar en primer lugar el empleo constante de la forma en plural, viruelas, para referirse a esta (ejemplo 1), en consonancia no solo con lo que ocurre en el México del siglo XVIII (Ramírez Luengo 2014: 190), sino -más general aún- con lo que constituye el uso habitual de la época en el mundo hispánico según los datos de CORDE; ; al mismo tiempo, el plural puede significar también el conjunto de pústulas que caracteriza a esta dolencia (ejemplo 2) ${ }^{9}$, de manera que no sorprende que el singular, que se emplea de manera mucho más escasa, presente el valor individualizado de 'pústula producida por la viruela' (DLE 2014: s.v. viruela), tal y como se aprecia en el ejemplo 3:

1. Harán un padrón de todos, desde los recién nacidos hasta los de catorce años poco más o menos, pues hasta los de esta edad no habrán tenido las viruelas, porque otro tanto tiempo haze que se padeció la última epidemia (p. 3).

2. Si las viruelas son muchas, chicas, y chatas, o si están entre sembradas con pintas negras [...], corren sumo peligro los enfermos (p. 8).

3. El primero que se apareciere en el pueblo con viruelas de buena calidad, se tomará con la punta de una lanzeta la materia de una viruela, de modo que quede bien embarrada (p. 4).

Junto a lo anterior, quizá sea especialmente importante señalar en este apartado la no aparición en el texto del latinismo variola para expresar la enfermedad o las pústulas que esta genera: en efecto, si bien en la documentación administrativa mexicana de esta misma época aparece con relativa frecuencia este término como voz hispánica integrada (Ramírez Luengo 2014: 191) ${ }^{10}$, en el caso de la obra guatemalteca que se está analizando este elemento está totalmente ausente, en lo

8 De acuerdo con los datos expuestos en Ramírez Luengo (2014: 190), el singular aparece en cuatro ocasiones durante el periodo 1701-1800, mientras que el plural, con 141 apariciones y el $97.24 \%$ del total, constituye la forma claramente privilegiada. Sería interesante analizar, por tanto, en qué momento comienza a imponerse en el habla el uso del singular, tal y como se registra actualmente en el mundo hispánico (CREA).

9 De hecho, dado que la enfermedad se manifiesta por medio de la aparición de múltiples pústulas, es de esperar que sea precisamente el plural el que de manera metonímica haga referencia a ambas cuestiones: el conjunto de pústulas y la dolencia en sí misma.

10 Como bien me señala Gómez de Enterría (comunicación personal), es probable que la presencia de este elemento en el español novohispano guarde relación con la lectura, por parte de los médicos, de la abundante bibliografía que sobre esta temática seguía redactándose en Europa en lengua latina. 
que en principio parece evidenciar una primera diferencia en este campo léxico entre las dos áreas del virreinato novohispano ${ }^{11}$.

Más allá de los términos utilizados para la enfermedad en sí, la necesidad de discriminar los distintos tipos que esta puede presentar obliga a emplear numerosos compuestos sintagmáticos que especifiquen de forma inequívoca de cuál de ellos se está hablando; en este sentido, si bien el corpus no resulta tan rico como el mexicano estudiado en Ramírez Luengo (2014), lo cierto es que ofrece también algunas muestras de tales compuestos que tienen que ver no tanto con las formas que adquiere la dolencia, sino más bien con otras circunstancias, tales como el tipo de viruela o la manera como se produjo el contagio. Por lo que se refiere a esta última cuestión, la introducción del proceso de variolización en Guatemala a partir de 1780 -precisamente por José Felipe Flores, el autor del texto analizado- explica la aparición en él de los compuestos viruelas naturales y su opuesto viruelas inoculadas (ejemplo 4), que hacen referencia "a las que el individuo muestra por contagio (...) frente a las más leves que se producen a resultas del proceso de la variolización” (Ramírez Luengo 2014: 198) y que parecen constituir las denominaciones generalmente aceptadas, tanto en la Nueva España como en la metrópoli (Ramírez Luengo 2014: 198).

4. Si las viruelas naturales son de buena calidad y benignas, como las inoculadas, se seguirá en todo la misma dieta de remedios y alimentos que se ha dicho (pp. 7-8).

En cuanto a los tipos de viruela, es necesario recordar ante todo la existencia de dos variante de la enfermedad que se diferencian fundamentalmente por su mortalidad: "la variola minor, con erupción característica pero con una reacción menos grave con una letalidad del 1\% o menos, y la variola major o viruela clásica, que eleva su letalidad en no vacunados del 15\% al 40\%" (Cortés Riveroll 2008: 20); por supuesto, una diferencia de tanta relevancia necesariamente tiene que ofrecer un reflejo léxico, que en el caso de este corpus adopta la forma de nuevos compuestos sintagmáticos: por lo que se refiere a la primera, José Felipe Flores emplea viruelas benignas y con mayor frecuencia viruelas de buena calidad (ejemplos 5, 6); en cuanto a la segunda, el texto ofrece tanto viruelas malas como sus sinónimos viruelas malignas y viruelas de mala calidad (ejemplos 7, 8, 9).

5. Se pegan las viruelas de uno que las padece de buena calidad a otro que no las tiene con el fin de que le salgan benignas (p. 3).

6. Si las viruelas naturales son de buena calidad y benignas, como las inoculadas, se seguirá en todo la misma dieta de remedios y alimentos que se ha dicho (pp. 7-8).

7. Este modo de cuidar a los enfermos con limpieza, y estos remedios [...], son los mejores para curar las viruelas malas (p. 9).

8. La calentura que se padece para que broten estas viruelas malignas es muy fuerte y acompañada de grande dolor de cabeza (p. 8).

11 Es necesario tener presente, con todo, que a la diferenciación geográfica mencionada se superponen también las distintas naturalezas tipológicas de los corpus considerados -documentación administrativa frente a tratado especializado-, lo que obliga a ser cautos a la hora de valorar exclusivamente lo diatópico como el factor que determina esta diferencia de uso; de hecho, es probable que lo diastrático tenga también una gran importancia en esta cuestión, habida cuenta de que el texto de Flores -que, como indica Gómez de Enterría (comunicación personal), bien podría englobarse dentro de las obras de divulgación- va destinado a receptores de muy diversos niveles de lengua que quizá no están familiarizados con el latinismo especializado. 
9. Las embarazadas que se hallan en los primeros meses se mantendrán absolutamente encerradas [...] pues se expondrían a contraer las viruelas naturales de muy mala calidad (p. 7) ${ }^{12}$.

De este modo, son varias las cuestiones que parece importante mencionar a partir de todo lo expuesto hasta el momento: por un lado, y como era de esperar, que la comparación de estos datos guatemaltecos con los del Altiplano mexicano permite señalar una notable coincidencia léxica entre ambas zonas geográficas, pues las dos comparten muchos de los términos presentes en el texto de Flores (Ramírez Luengo 2014: 193-194) ${ }^{13}$; por otro, es también destacable la aparición de alternancias como viruelas malas/malignas/de mala calidad o benignas/de buena calidad, esto es, de sintagmas formalmente distintos que poseen el mismo significado y que por ello se pueden interpretar como ejemplos de voces fluctuantes, cuya aparición en este discurso, previa "a la acuñación definitiva de los términos médicos, es una de las características de estos vocabularios” debido "a su novedad o al uso inseguro que el especialista hace de las nuevas voces ante la recepción de estas" (Gómez de Enterría 2012a: 71); finalmente, es así mismo interesante señalar la ausencia en la obra del chiapaneco de los numerosos compuestos que hacen referencia a las distintas clases de erupción que muestra la enfermedad -tales como viruelas fatuas, viruelas discretas, viruelas confluentes o viruelas cristalinas (Ramírez Luengo 2014: 195-196)-, algo que muy probablemente guarde relación con el ya mencionado carácter práctico de la Instrucción y sobre todo con el público, no necesariamente especialista, a quien va dirigida, circunstancias ambas que parecen favorecer un uso restringido de los vocablos especializados menos difundidos o de más difícil comprensión para el lego ${ }^{14}$.

Por otro lado, son también destacables los vocablos con los que José Felipe Flores se refiere en su obra tanto al proceso del contagio de la enfermedad en sí como a las fases por las que esta atraviesa desde este momento hasta su definitiva curación, los cuales resultan de gran interés no solo por su abundancia, sino muy especialmente porque en ambos casos se observa la alternancia de voces cultas con otras que -de manera más o menos imprecisa- se pueden considerar populares/ coloquiales y que forman parte, por tanto, del "vocabulario con el que el profano hace referencia a la experiencia de la enfermedad y/o la medicina en su vida cotidiana" (Ramírez Luengo 2015: $296)^{15}$.

12 Aunque el ejemplo permite interpretar que viruelas de mala calidad supone un mero sintagma ocasional y no una denominación para la viruela major, el hecho de que se emplee con cierta frecuencia en tratados médicos dieciochescos y documentación administrativa novohispana de esta misma época (Ramírez Luengo 2014: 193) inclina a considerar que se trata de un compuesto más, sinónimo de viruelas malignas y paralelo a viruelas de buena calidad.

13 De hecho, México parece ofrecer una mayor variedad al respecto, dado que se descubren también viruelas sin malicia (Ramírez Luengo 2014: 193), si bien la diferente extensión de los corpus utilizados dificulta una comparación de carácter cuantitativo como la que se acaba de esbozar.

14 De hecho, es probable que esta intención de Flores de adecuarse a sus potenciales lectores explique por qué, al hablar de este asunto, se decanta por un sintagma como viruelas sembradas con pintas (p. 12), no solo de carácter descriptivo, sino además compuesto con una voz popular como pinta (Ramírez Luengo 2015: 301-302).

15 Este tipo de vocabulario -que Gómez de Enterría y Gallardo (2010: 58) denominan “el léxico de los pacientes”"muy difícilmente tiene cabida en los tratados científicos, los textos docentes o todas aquellas obras que reflejan el discurso médico académico y especializado" (Ramírez Luengo 2015: 296); por supuesto, el hecho de que en este caso no sea así se debe a las peculiares características de esta obra, tanto en lo que se refiere a sus potenciales receptores como a su carácter eminentemente práctico, según se ha indicado con anterioridad. Para una reflexión sobre cómo entender y documentar esta peculiar terminología médica, véase lo indicado en Ramírez Luengo (2015: 300-306). 
En el caso del campo léxico del contagio, la Instrucción presenta una serie de sinónimos que hacen referencia a esta cuestión y que evidencian la convivencia, junto a voces de carácter más o menos culto -al estilo de contagiar o contaminar (ejemplo 10), presentes con este valor especializado ya en Autoridades (RAE 1979: s.v. contagiar, contaminar) ${ }^{16}$-, de otras que muy probablemente pertenecen al ámbito popular y que por ello se pueden englobar sin dificultades dentro del ya mencionado "léxico de los pacientes", al estilo de los usos metafóricos de pegar o -con más dudas- pasar (las viruelas) (ejemplos 11 y 12$)^{17}$ :

10. Inmediatamente que se descubre en el pueblo algún muchacho con viruelas o que, por estar muy cerca de otro pueblo, le ha contagiado [...], es casi imposible que dejen de contaminar las viruelas (p. 3).

11. La inoculación de las viruelas es una operación por la cual se pegan las viruelas de uno que las padece de buena calidad a otro que no las tiene (p. 3).

12. Si se practica lo que queda referido, seguramente las viruelas no pasarán de los pueblos (p. 10).

Esta preferencia por el empleo de voces de uso general para hacer referencia a conceptos del ámbito de la medicina se hace aún más evidente en el caso de la nomenclatura que Flores aplica a las fases de la enfermedad; por lo que se refiere a estas, señala Cortés Riveroll (2008: 20) que, tras una primera etapa con fiebre, malestar general o cefalalgia, el enfermo comienza a mostrar unas pequeñas erupciones en la cara que se extienden posteriormente por el cuerpo y pasan a convertirse en pústulas, las cuales terminan por caer en la tercera o cuarta semana, momento en el que el paciente se puede dar por curado. Como es de esperar, si todos estos momentos reciben en la medicina dieciochesca diversas denominaciones especializadas -del estilo de principio, aumento, invasión, supuración o declinación (Ramírez Luengo 2014: 205-206)-, lo mismo va a suceder en la obra analizada, aunque en ella es posible señalar dos diferencias de interés respecto a otros tratados novohispanos o a la documentación administrativa de este mismo origen: por un lado, el establecimiento de tres fases y no cuatro, equiparables a la aparición de las pústulas, su generalización y su desaparición; por otro, la decidida apuesta por el uso de voces hispánicas comunes como términos de especialidad -en concreto brotar/salir (las viruelas), madurar (las viruelas) y secarse (las viruelas)-, las cuales resultan, además, claramente descriptivas de los diversos procesos (ejemplos 13-16):

13. De este modo se les asistirá por tres o cuatro dias, hasta que acaben de brotar las viruelas (p. 6). 14. Si acomete a alguno de los chicos, cuando les comienzan a salir las viruelas, algunos movimientos como de perlesía, no hay que tener cuidado (p. 6).

15. Se les volverá a sangrar cuando, después de haber brotado las viruelas, la calentura vuelve a encenderse, para que maduren (p. 8).

16 Como antónimo culto de ambos vocablos, en el texto se descubre también la forma preservarse (p. 14), que presenta el significado de 'evitar el contagio'.

17 Conviene señalar, una vez más, que estos usos en modo alguno suponen un rasgo original del médico chiapaneco, sino un claro aprovechamiento de las posibilidades existentes ya en la lengua de la época, tal y como demuestra la aparición de estos valores de naturaleza médica en el Diccionario de Autoridades, tanto en el caso de pegar ("vale también comunicar una cosa a otra. Comúnmente se dice de las enfermedades contagiosa"; RAE 1979: s.v. pegar) como en el de pasar ("Vale asimismo extenderse o comunicarse de unos en otros, o pegarse alguna cosa, como se dice de los contagios"; RAE 1979: s.v. pasar). 
16. Finalmente cuando las viruelas se han secado y comienzan a caer las costras, se les dará agua de tamarindo (p. 7).

Al mismo tiempo, el texto analizado muestra también una clara preferencia por el empleo de voces coloquiales a la hora de referirse a quien ha contraído la viruela, si bien en este caso tales voces populares conviven con otras de carácter más especializado, todo lo cual configura nuevamente un conjunto de términos sinónimos que, en un claro ejercicio de variatio, emplea el doctor chiapaneco a lo largo de su obra para referirse al mismo referente. Por lo que se refiere a los primeros, cabe mencionar los muy generales -tanto en lo que se refiere a su significado como a su uso en el Setecientos- enfermo o contagiado (ejemplos 17, 18), así como apestado (ejemplo 19), estos dos últimos empleados siempre como adjetivos:

17. Este modo de cuidar a los enfermos con limpieza y estos remedios [...] son los mejores para curar las viruelas (p. 9).

18. Que, en los pueblos no contagiados, el teniente, los justicias y principales y demás vecinos tengan sumo cuidado, en las entradas, de atajar a todos (p. 10).

19. Que, en los pueblos y haciendas apestadas, se mande bajo de graves penas no salga ninguno, y particularmente muchachos, para ninguna parte (p. 9).

Frente a estos elementos, es interesante mencionar también aquellas otras denominaciones que muy probablemente pertenecen de manera preferente -pero no exclusiva- al registro médico y que se crean por derivación a partir del nombre de la enfermedad, virueliento y virolento (ejemplos 20, 21) $)^{18}$ :

21. Que en estos pueblos no se ponga ningún virueliento en el Cabildo (pp. 9-10).

22. Quita toda esperanza de que por ahora sea practicable con algún éxito el medio de aislar a los primeros virolentos (pp. 13-14).

Por lo que se refiere a este último vocablo, se trata de un término ampliamente documentado durante el siglo XVIII tanto en España como en América, y que aparece no solo en tratados especializados -a manera de ejemplo, Boix y Moliner (1711: 128) o García Hernández (1747: 177)- y documentación administrativa relacionada con la práctica médica, sino también en contextos no científicos como el Diccionario de Autoridades o determinadas obras de carácter literario (Ramírez Luengo 2014: 200), todo lo cual evidencia un empleo relativamente amplio en la época, que se extiende más allá del discurso propio de la medicina.

En contraste con esto, virueliento parece mostrar una distribución mucho más escasa: al igual que en el caso de viruelento (Ramírez Luengo 2014: 201-202) -con el que sin duda guarda relación ${ }^{19}-$, este derivado de viruela está ausente no solo de los grandes corpus diacrónicos (CORDE,

18 Es necesario señalar que, aunque ambos vocablos aparecen en el impreso que se está analizando, lo cierto es que no lo hacen en la misma parte, dado que virueliento se encuentra en el texto de la Instrucción, mientras que virolento hace acto de presencia en uno de los memoriales adjuntos, firmado por Ignacio Guerra.

19 Aunque en principio virueliento se podría considerar una simple errata por viruelento, el hecho de que -como se dirá a continuación- aparezca en varios impresos invalida tal interpretación; esta idea se ve avalada, además, por 
CORDIAM, LEXHISP), sino también de las distintas ediciones del DRAE (NTLLE) o de la obra lexicográfica de Terreros (1987[1786]), y tan solo hace acto de presencia de forma ocasional en diferentes textos americanos del Ochocientos relacionados con la medicina, tanto mexicanos como peruanos y cubanos (González 1882: 66; Valdizán 1957: 20; Romay 1844: 220); se puede considerar, por tanto, un término restringido diatópicamente al Nuevo Mundo cuya aparición es necesario adelantar al menos a los últimos años de la Centuria Ilustrada y cuyo empleo junto a viruelento, virolento y varioloso constituye una muestra específicamente americana de la fluctuación formal que, como bien señala Gómez de Enterría (2012a: 71), resulta frecuente en el vocabulario médico de esta época.

4. Así pues, el análisis del léxico relacionado con la viruela que se descubre en la Instrucción del doctor chiapaneco José Felipe Flores permite extraer ya algunas conclusiones de interés para la más profunda comprensión de la configuración del vocabulario médico dieciochesco relacionado con esta dolencia no solo en Guatemala, sino también en América y -de manera más amplia- en el mundo hispánico en general.

A este respecto, quizá lo primero que se hace necesario señalar es el alto nivel de coincidencia detectado entre los ámbitos geográficos mencionados, que en este campo léxico concreto atañe a los nombres empleados, por ejemplo, para referirse a los distintos subtipos de viruela o al contagio, y que sin duda tiene su origen en la tendencia a la uniformidad que caracteriza al vocabulario científico, pero también "en la existencia de un bagaje teórico y unas lecturas comunes, así como en las mutuas relaciones de distinto tipo que se dan entre los dos continentes" (Ramírez Luengo 2014: 208); con todo, un análisis más detallado demuestra que esta esencial uniformidad convive así mismo con lo que parecen ser preferencias de uso diatópicamente determinadas, tales como la ausencia de variola y varioloso en la obra y quizá en la propia Guatemala -en contraste, por ejemplo, con su empleo frecuente en los textos mexicanos de esta misma época- o la aparición en ambas zonas de virueliento/viruelento, esto es, de elementos que parecen emplearse de forma exclusiva en los territorios americanos ${ }^{20}$ y que contribuyen, así, a dotar de personalidad propia al discurso médico del Nuevo Mundo.

Junto a lo anterior, resulta también destacable en este corpus la aparición relativamente abundante de términos considerados populares que Gómez de Enterría y Gallardo (2010: 58) engloban dentro del "léxico de los pacientes" y que constituyen ejemplos de los vocablos "con que los no especialistas se refieren a la enfermedad y a sus consecuencias" (Ramírez Luengo 2015: 300), muchas de las cuales no suponen sino usos metafóricos especializados que, para referirse a conceptos médicos, se forman a partir de voces cotidianas, tal y como demuestra el empleo de pasar y pegarse para referirse al contagio de la viruela o los diferentes términos (brotar/salir, madurar y secar) con los que Flores denomina las distintas fases de la enfermedad; a este respecto, no cabe duda de que tanto el carácter eminentemente práctico que presenta la Instrucción como sobre todo el público al que esta va dirigida -diferente personal de la administración civil y religiosa, no necesariamente especialistas en el área de la medicina- explican la aparición de unidades léxicas

su aparición como "palabra castiza” en la obra prescriptiva del guatemalteco Batres Jáuregui (1892: 548), lo que parece confirmar su empleo en el país durante el siglo XIX.

20 Constatación que, dicho sea de paso, permite hablar de americanismos léxicos en un ámbito en principio tan poco propicio para ello como es el léxico de la medicina o de la ciencia en general. 
como estas en un texto de carácter técnico ${ }^{21}$, lo que una vez más demuestra la estrecha relación que, en el marco de la medicina dieciochesca, se establece entre la finalidad de una obra específica y el vocabulario que se utiliza en ella.

En conclusión, parece indiscutible que, tanto por la amplitud de temáticas tratadas como por la importancia histórica de su autor y la riqueza léxica que atesora, la Instrucción sobre el modo de practicar la inoculación de la viruelas constituye un corpus de primera importancia para el análisis del vocabulario médico de la Guatemala del siglo XVIII cuyo interés está muy lejos de agotarse con esta primera aproximación a la terminología relacionada con la viruela; no queda, pues, sino seguir estudiando el texto del doctor José Felipe Flores, que sin duda tiene aún mucho que ofrecer para el mejor conocimiento de un campo tan ignorado por el momento como son las voces que, en plena Centuria Ilustrada, emplean en su práctica profesional los galenos del antiguo Reino de Guatemala.

\section{Referencias bibliográficas}

Álvarez Sánchez, A. (2016). Patronazgo y educación. Los proyectos y la fundación de la Real Universidad de San Carlos de Guatemala (1619-1687). Ciudad de México: Universidad Nacional Autónoma de México. Asturias, F. (1902). Historia de la medicina en Guatemala. Ciudad de Guatemala: Tipografía Nacional. Aznar López, J. (1998). José Felipe Flores, científico chiapaneco de la Ilustración hispanoamericana. Tuxtla Gutiérrez: Coneculta Chiapas.

Barona Vilar, J. L. (2019). José Felipe Flores. In Real Academia de la Historia (org.), Diccionario biográfico electrónico. <http://dbe.rah.es/ biografias/19369/jose-felipe-flores>

Batres Jáuregui, A. (1892). Vicios del lenguaje y provincialismos de Guatemala. Ciudad de Guatemala: Tipografía Nacional.

Belaubre, C. (2019). Flores, José. In AFEHC. Asociación para el fomento de los estudios históricos en Centroamérica. Diccionario. <http://www.afehc-historia-centroamericana.org/index_action_fi_aff_id_1227. html>

Boix y Moliner, M. M. (1711). Hippocrates defendido. Madrid: Matheo Blanco.

[CORDE]: Real Academia Española (2019). Banco de datos CORDE. Corpus diacrónico del español. <http:// www.rae.es>

[CORDIAM]: Academia Mexicana de la Lengua (2019). Corpus Diacrónico y Diatópico del Español de América. <http://www.cordiam.org/>

Cortés Riveroll, J. G. R. (2008). Inicio de la Vacunación en la ciudad de Puebla. 1804-1814 (Cuadernos de Trabajo, 30). Xalapa: Universidad Veracruzana.

21 Aunque resulta difícil de demostrar, es probable que también la conciencia estética de José Felipe Flores le lleve a utilizar en ocasiones estos elementos como un recurso retórico de variatio que le permita evitar la excesiva repetición de un vocablo; esta motivación parece ser especialmente evidente en pasajes donde le es necesario referirse de manera continuada a un mismo referente -por ejemplo a la variola major-, en los que opta por utilizar de manera indistinta los diversos sinónimos con que se expresa el mismo concepto: viruelas malas, viruelas malignas y viruelas de mala calidad (pp. 7-9). 
[CREA]: Real Academia Española (2019). Corpus de referencia del español actual. <http://www.rae.es/recursos/banco-de-datos/crea>

[DLE]: Real Academia Española (2014). Diccionario de la Lengua Española. Madrid: Espasa-Calpe.

Galich, L. F. (1982). El doctor José Felipe Flores y la expedición de la vacuna alrededor del mundo. Anales de la Sociedad de Geografía e Historia de Guatemala, 56, 137-157.

García Hernández, F. (1747). Tratado de fiebres malignas. Madrid: Miguel Francisco Rodríguez.

Gómez de Enterría, J. (2012a). El vocabulario médico de los novatores en el siglo XVIII. In M. T. García Godoy (Ed.), El español del siglo XVIII. Cambios diacrónicos en el primer español moderno (pp. 55-81). Berna: Peter Lang.

. (2012b). El vocabulario de la medicina del siglo XVIII a través de las traducciones de los hermanos Juan y Félix Galisteo Xiorro. In E. Montero (Ed.), Actas del VIII Congreso Internacional de Historia de la Lengua Española (vol. 2) (pp. 1405-1422). Santiago de Compostela: Meubook.

- (2013). Higiene y salud en las traducciones médicas del francés al español durante el siglo XVIII. Panace@. Revista de medicina, lenguaje y traducción, 14, 38, 287-295.

. (2014). El vocabulario de las fiebres epidémicas en el español del siglo XVIII. España y México. In J. L. Ramírez Luengo, \& E. P. Velásquez Upegui (Eds.), La historia del español hoy. Estudios y perspectivas (pp. 199-216). Lugo: Axac.

Gómez de Enterría, J.; \& Gallardo, N. (2010). Las versiones de medicina y botánica y la nueva terminología científica en el siglo XVIII. Cuadernos del Instituto de Historia de la Lengua, 4, 55-75.

González, J. E. (1882). Un punto de higiene. Monterrey: Imprenta del Gobierno.

Gutiérrez Rodilla, B. M. (1993). Los términos relacionados con la medicina en el Diccionario de Autoridades. Boletín de la Real Academia Española, 73, 260, 463-512.

- (1994-5). Construcción y fuentes utilizadas para los términos médicos en el Diccionario de Autoridades. Revista de Lexicografía, 1, 149-162.

. (1996). El léxico de la medicina en el diccionario de Esteban Terreros y Pando. In A. Alonso González et alii. (Eds.), Actas del III Congreso Internacional de Historia de la Lengua Española (vol. 2) (pp. 1327-1342). Madrid: Arco/Libros.

Hernández Pérez, J. S. (2010). Medicina y salud pública: su difusión a través de la Gaceta de Guatemala (1797-1804). EÄ. Revista de Humanidades Médicas \& Estudios Sociales de la Ciencia y la Tecnología, 2, 2, $1-34$.

[LEXHISP]: Boyd-Bowman, P. (2003). Léxico hispanoamericano. 1493-1993. Nueva York: Hispanic Seminary of Medieval Studies. <http://textred.spanport.lss.wisc.edu>

López Piñero, J. M. et alii. (1992). Bibliographia Medica Hispánica, 1415-1850, III. Libros y folletos, 17011800. Valencia: Universidad de Valencia.

Martínez Durán, C. (2010). Las ciencias médicas en Guatemala: origen y evolución. Ciudad de Guatemala: Editorial Universitaria.

[NTLLE]: Real Academia Española (2019). Nuevo Tesoro Lexicográfico de la Lengua Española. <http://ntlle. rae.es/ntlle/SrvltGUILoginNtlle>

Parcero Torre, C. (2011). El discurso científico en pareceres médicos en Colombia, en el siglo XVIII. Cuadernos de la ALFAL, 2, 196-203.

Ramírez Luengo, J. L. (2014). Un aporte a la historia del léxico médico en América: el vocabulario de la viruela en la Nueva España dieciochesca. Anuario de Letras. Lingüística y Filología, 2, 1, 181-214. 
populares en la denominación de las enfermedades. Cuadernos Dieciochistas, 16, 291-310.

. (2015b). Aproximación al léxico de la medicina en el Buenos Aires del siglo XVIII. In J. M. García Martín (Ed.), Actas del IX Congreso Internacional de Historia de la Lengua Española (vol. 2) (pp. 2151-2164). Madrid/Frankfurt: Iberoamericana/Vervuert.

- (2019). Los indigenismos en el Florilegio medicinal de Juan de Esteyneffer (1712). Nuevas Glosas. Estudios de Literatura y Lingüística, 1 (en prensa).

. (2020). Explicar lo desconocido: la incorporación discursiva de los indigenismos en el Florilegio Medicinal de Juan de Esteyneffer. Nueva Revista de Filología Hispánica, 68, 255-268.

Real Academia Española (1979). Diccionario de Autoridades (edición facsímil de la de 1726-1739). Madrid: Gredos.

Rodríguez, M. E. (1990). Dr. José Felipe Flores, el primer protomédico de Guatemala. Boletín de la Sociedad Mexicana de Historia y Filosofía de la Medicina, 13, 111-123.

Rodríguez, M. E. (2001). La viruela. In M. E. Rodríguez Pérez, \& X. Martínez Barbosa (Coords.), Historia general de la medicina en México, IV. Medicina novohispana. Siglo XVIII (pp. 129-134). México DF: Academia Nacional de Medicina / UNAM.

Romay, T. (1844). Elogio del Doctor Don Eusebio Valli. Memorias de la Sociedad Económica de La Habana (vol. XVIII) (pp. 218-224). La Habana: Imprenta del Gobierno y Capitanía General.

Smith, M. M. (1974). The Real Expedición Marítima de la Vacuna in New Spain and Guatemala. Transactions of the American Philosophical Society, 64, 1, 1-74.

Terreros, E. de (1987 [1786]). Diccionario castellano con las voces de ciencias y artes y sus correspondientes en las tres lenguas francesa, latina e italiana. Madrid: Arco/Libros.

Valdizán, H. (1957). Diccionario de medicina peruana, III. Lima: Universidad Nacional Mayor de San Marcos. 
